\title{
Collimated Beam FMCW Radar for Vital Sign Patient Monitoring
}

\author{
A. Prat, Member, IEEE, S. Blanch A., Aguasca, Member, IEEE, J. Romeu, Fellow, IEEE, A. \\ Broquetas, Member, IEEE
}

\begin{abstract}
Patient monitoring of vital signals such as breathing rhythm and heart beat rate can be done remotely by the use of a radar system. This approach is advantageous since it does not require any contact with the patient. Obviously contactless monitoring results in a more comfortable situation for the patient, and in occasions it is almost mandatory as in the case of heavy burnt or newborn patients. Moreover, additional information such movement patterns are also available. A $120 \mathrm{GHz} F \mathrm{FCW}$ radar is described with special focus on the design, construction and testing of a specific reflector antenna for the system. The system is based on a commercial radar chipset that includes its own antennas. The challenge has been to design the optimum reflector and to build it and test it in a cost effective way. The reflector has been $3 \mathrm{D}$ printed and a near-field testing technique has been implemented to assess its performance. The results show that the system is able to measure the vital signs at distances beyond one meter.
\end{abstract}

Index Terms-Biomedical Telemetry, FM radar, Millimeter wave radar, reflector antennas.

\section{INTRODUCTION}

$\mathrm{R}$ EMOTE contactless measurement of vital signs such as breathing rhythm and heart beat rate can improve patient attention in clinical environments. In [1] a thorough review of different technology approaches and their performances in clinical environments is presented. Radar technologies are appealing, but the reported systems operate at low frequencies. In general, they are bulky and with high susceptibility to environmental conditions such as multiple reflections. Early proposals of remote monitoring systems based in radar measurements of thorax movements can be found in [2] and [3]. More recently Doppler measurements to monitor vital signs with continuous wave $(\mathrm{CW})$ radars have been presented at frequencies of 4, 16 and $34 \mathrm{GHz}$ [4][5][6]. In this work, we propose a real-time, contactless and non-obstructive system for heartbeat and breathing rate monitoring operating in the ISM band of $120 \mathrm{GHz}$. The system is an FMCW radar that allows making accurate range measurements and the measurement of small displacements as the ones produced in the chest by the heartbeats. The use of an FMCW radar as compared to CW radars allows to have accurate range positioning of the test body. In this way it is possible to clearly identify return signals

Manuscript received Feb XX, 2018; date of current version nulldate. This work was supported in part by the Spanish Inter-Ministerial Commission on Science and Technology (CICYT) under projects TEC201678028-C3-1P,TIN2014-55413-C2-1-P, and TEC2017-85244-C2-2-P and, by FEDER and the Unidad de Excelencia Maria de Maeztu MDM-2016-0600, which is coming from the desired target and discard those undesired returns coming from the uncontrolled environment. Also the small wavelength of the carrier frequency allows the design of small electromagnetic wave focusing elements. In this way a small dimension non-intrusive device can be designed with high immunity to interferences of co-sited equipment and robust against unwanted reflections from the uncontrolled environment. The paper has a system approach and it gives special attention to the design of the specific antenna system. The principles of operation of an FMCW radar are also briefly presented to understand the operation of the system.

The rest of the paper is organized as follows. Section 2 describes the overall architecture of the system. In Section 3 the design, construction and testing of the reflector used in the system is explained. In Section 4 the experimental results are presented. Finally, the conclusions are stated in Section 5.

\section{SYSTEM DESCRIPTION}

The functionality of the system is shown in Fig. 1. The goal is to design an FMCW radar system placed at a distance of 1.5 $\mathrm{m}$ above a patient and being able to measure breathing rhythm and heart beat rate from the displacement of the chest. The system has to be of reduced dimensions, non-invasive and being able to operate in uncontrolled environments.

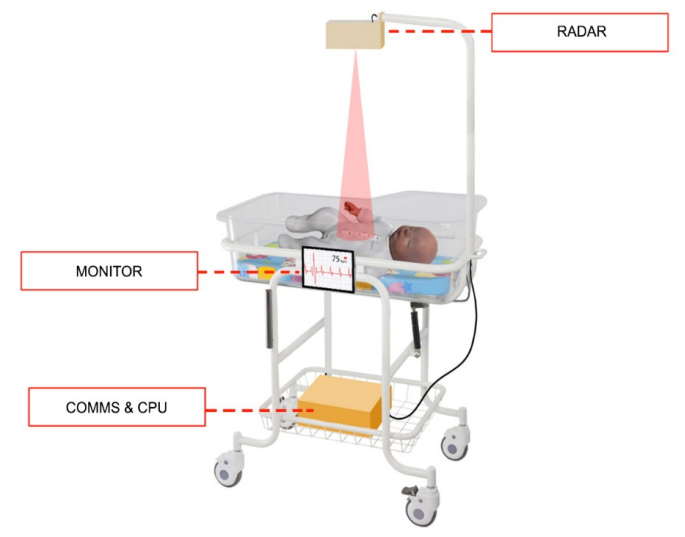

Fig. 1. Mockup of the system configuration.

financed by the Agencia Estatal de Investigación, Spain. A. Prat, S. Blanch, A. Aguasca, J. Romeu, A. Broquetas, are with the CommSensLab, Department of Signal Theory and Communications, Universitat Politècnica de Catalunya (UPC), 08034 Barcelona, Spain (e-mail: romeu@tsc.upc.edu). 
The range resolution achieved with the FMCW configuration combined with the narrow antenna beamwidth provides excellent spatial selectivity of the patient area of interest with respect surrounding objects. The basic principles of FMCW radar can be found in general and specialized radar textbooks [7],[8], but to introduce notation that will be used later a short mathematical derivation of FMCW detection is also presented here. The basic scheme of an FMCW radar is shown in Fig. 2.

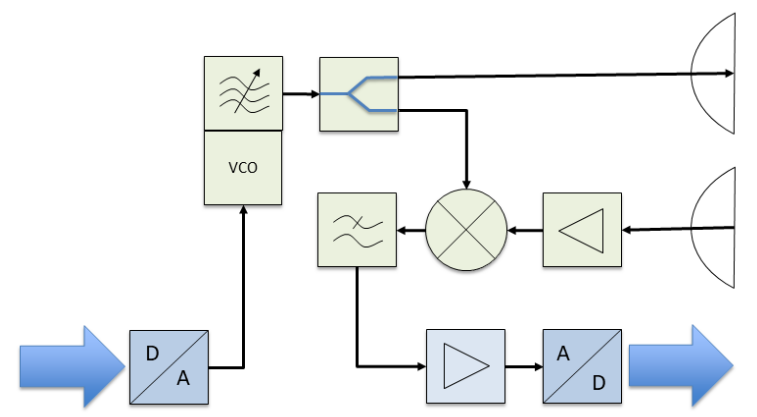

Fig. 2. Basic scheme of an FMCW radar.

The transmitted signal changes its frequency linearly with time as shown in Fig. 3 and it can be represented as a complex exponential as in (1). Where $\omega=2 \pi f$, being $f$ the starting frequency, and $\alpha$ is the chirp rate defined in (2).

$$
\begin{gathered}
s_{t}=e^{-j\left(\omega t+\frac{\alpha}{2} t^{2}\right)} \\
\alpha=\frac{2 \pi\left(f_{\text {end }}-f_{\text {start }}\right)}{T_{\text {sweep }}}=2 \pi \frac{B}{T_{\text {sweep }}}
\end{gathered}
$$

If a target is at a distance $\mathrm{R}$, the radar will receive the echo signal with a round trip delay $\tau$ as in (3). Where $\mathrm{c}$ is the speed of light, and the received signal can be represented as in (4).

$$
\begin{gathered}
\tau=\frac{2 R}{c} \\
S_{r}=e^{-j\left(\omega(t-\tau)+\frac{\alpha}{2}(t-\tau)^{2}\right)}
\end{gathered}
$$

As shown in Fig. 2, in a FMCW radar the detected signal is the result of the beating between the transmitted and received signal. Its time variation can be expressed as:

$$
s(t)=\cos \left(\alpha \tau t+\omega \tau-\frac{\alpha \tau^{2}}{2}\right)
$$

From (5) the detected signal corresponding to a target at a distance $\mathrm{R}$ will have a frequency shown in (6), corresponding to the first term of (5), and a linear phase term shown in (7), corresponding to the second term in (5).

$$
\begin{gathered}
f(R)=\frac{\alpha R}{\pi c} \\
\varphi(R)=\omega \tau=\frac{\omega}{c} 2 R=4 \pi \frac{R}{\lambda}
\end{gathered}
$$

The third term in (5) is a quadratic phase term, also depending on the distance that for short distances can be neglected in front of the linear phase term. The linear phase term shows that for changes in range of the target of half a wavelength the phase term will change $2 \pi$. Therefore, the knowledge of the frequency of the detected signal provides a measure of range of the target, while the signal phase evolution along time shows the target radial motion with sub-millimeter precision as evidenced in civil engineering applications of FMCW radar [9]. As long as the small movements produced on the chest due, for example, to the heartbeat, are significant in terms of the wavelength that will cause phase changes on the detected signal that can be measured.

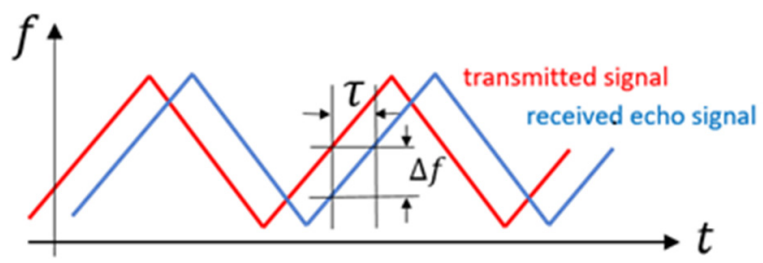

Fig. 3. Ranging with an FMCW radar

The front-end of the radar used for the prototype is based on a compact sensor developed by Silicon Radar [10]. The chip includes both the transmitting and receiving antennas and the rest of the high frequency electronics. The radar is able to operate at a center frequency of $122 \mathrm{GHz}$ with a bandwidth of up to $7 \mathrm{GHz}$. The nominal output power is $-3 \mathrm{dBm}$. Since the antennas on the chip do not provide the collimated beam required for the desired application it is necessary to design an optimum parabolic reflector that can be easily integrated. In section III a detailed description of the design, construction and testing of a parabolic reflector is presented.

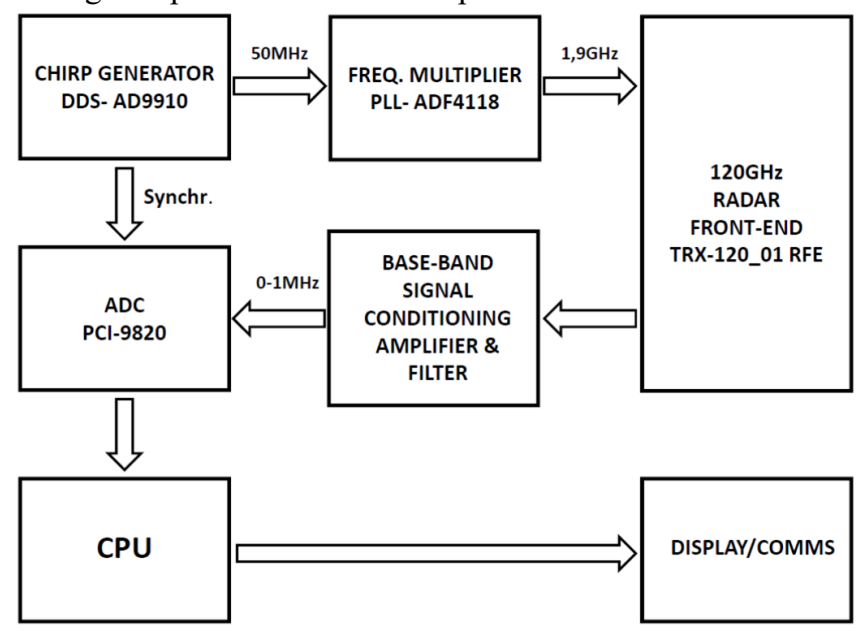

Fig. 4. Block diagram of the FMCW radar system.

Apart from the radar front-end, and the reflector antenna the system requires additional elements to operate. The radar front end has an internal VCO that provides a sample of the transmit frequency divided by 64 . Therefore, it is necessary to externally 
include the necessary elements to produce the chirp signal. Below are described the rest of the parts of the system as shown in Fig. 4: 1) DDS/PLL. It generates a triangular ramp signal together with the necessary time references to synchronize the system (trigger and sampling clock), it controls the VCO in the RF frond end and closes the PLL to have an accurate frequency control. The PLL has been designed to minimize the ramp nonlinearities. 2) Signal Conditioning: It amplifies and filters the I/Q signals received by the radar front-end using low-noise amplifiers 3) ADC: It samples the signals to be able to be processed by the computer, 4) CPU/Processing of the signal based on Fourier Transform to obtain range resolution and extraction of the vital signals of interest.

Despite the large signal bandwidth, the radar range resolution is not sufficient to detect the small displacements of the detected object (i.e respiration chest movement or heartbeat chest deformation). However, the phase of the range cell peak corresponding to the patient sensed spot provides the body surface radial motion with a sensitivity of $3.47 \mu \mathrm{m} /{ }^{\circ}$ at $120 \mathrm{GHz}$ which is enough to monitor respiratory and cardiac functions. In this way, the processing includes the following steps: A) Fast Fourier Transform (FFT) of the received signal to obtain range resolution, B) Peak detection in a preselected range interval to locate the sample corresponding to the patient observed spot, this allows target tracking in the event the patient moves and to discard unwanted reflections coming from objects at other distances C) Obtain the echo phase from the target peak complex sample. D) Phase-Unwraping to avoid $+/-\pi$ phase jumps and phase to range conversion according to (7). E) LowPass and Band-Pass filtering of unwrapped phase signal to separate respiration and heart contributions to observed motion. F) Calculation and display of respiration and heart beat frequencies and trigger alarms in case of apnea, tachycardia, cardiac arrest and other abnormalities.

\section{REFLECTOR DESIGN AND CONSTRUCTION}

For the detection of small chest movements, it is required to have a collimated beam of about $10 \mathrm{~cm}$ diameter for distances of the order of $1.5 \mathrm{~m}$. As said before, the radar is based on an integrated $120 \mathrm{GHz}$ transceiver made by Silicon Radar model TRX_120_001 [10]. The transceiver includes an integrated set of transmitting and receiving antennas. As shown in Fig. 5 each set of antennas is a 2 by 2 array of microstrip patch antennas. Assuming a uniform excitation of the elements of the array, a straightforward analysis shows that the resulting radiation pattern has approximately $45^{\circ}$ half-power beam widths in both planes. This wide radiation pattern results in unwanted radar interferences created by other objects around the patient and low Signal-to-Noise ratio. Thus the radar patient observation has been substantially improved with the design of a specific beam-collimating element that is minimally intrusive with the radar operation.

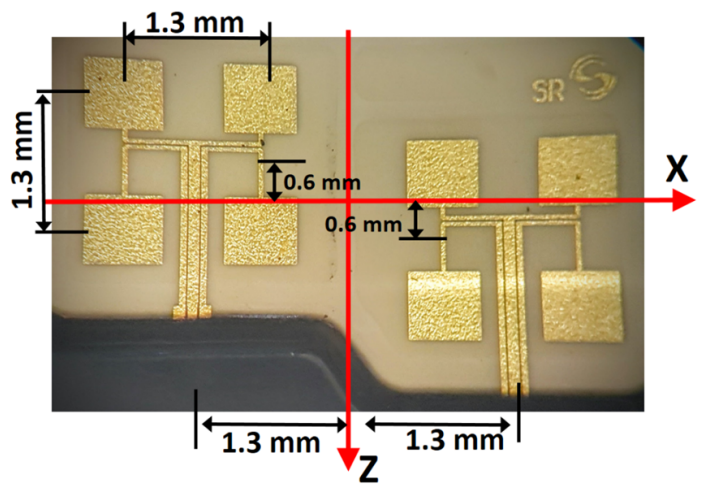

Fig. 5. Antennas of the radar transceiver. One array is the transmitter and the other the receiver.

Two simple collimating elements are possible, a lens or a parabolic reflector. The choice of the lens is discarded because of the losses that would be introduced, both in the dielectric material of the lens and also due to the mismatch in the lens-air interfaces. In consequence, the parabolic reflector option is preferred. To avoid blocking losses and simplify the assembling procedure of the set-up a 90 degrees offset reflector geometry is chosen. The first step is to assess the footprint that a $10 \mathrm{~cm}$ diameter aperture would produce on the target. At a frequency of $120 \mathrm{GHz}(\lambda=2.5 \mathrm{~mm})$ the Fraunhofer or far-field distance of a $10 \mathrm{~cm}$ aperture diameter reflector is at $8 \mathrm{~m}$. Therefore, our zone of interest is within the near-field region of the reflector. Before proceeding with any further electromagnetic analysis and in order to assess the footprint that a $10 \mathrm{~cm}$ diameter reflector produces on a target at a distance of $1.5 \mathrm{~m}$ a Gaussian beam propagation approach can be used to estimate the collimating effect of the reflector. The aperture has 40 wavelengths of diameter and it is assumed a Gaussian field distribution on the aperture of the type:

$$
U=e^{-\left(\frac{r}{w_{o}}\right)^{2}}
$$

where $\mathrm{r}$ is the radial distance from the aperture center and $\mathrm{w}_{0}$ is the waist radius of the field distribution on the aperture. The waist radius $\mathrm{w}$ of the field distribution as a function of the distance $\mathrm{z}$ from the aperture is given in eq. (9) and $\mathrm{zr}$ is the Rayleigh range given by (10) [11].

$$
\begin{gathered}
\frac{w(z)}{w o}=\sqrt{1+\left(z / z_{r}\right)^{2}} \\
z_{r}=\pi \frac{w_{o}^{2}}{\lambda}
\end{gathered}
$$

Where $\lambda$ is the wavelength. Finally, for a given taper $\tau$ in the aperture field distribution expressed in $\mathrm{dB}$ as positive number the field waist in the aperture can be approximated as:

$$
w_{o}^{2}=\frac{r_{a}^{2}}{\tau} 20 \log (e)
$$

where $r_{a}$ is the radius of the aperture. Fig. 6 shows the waist radius as a function of the distance to the aperture for different values of the aperture field taper. It is observed that at a distance of $1.5 \mathrm{~m}$ the waist radius is for all the cases of the order of $5 \mathrm{~cm}$, which is the design goal. 


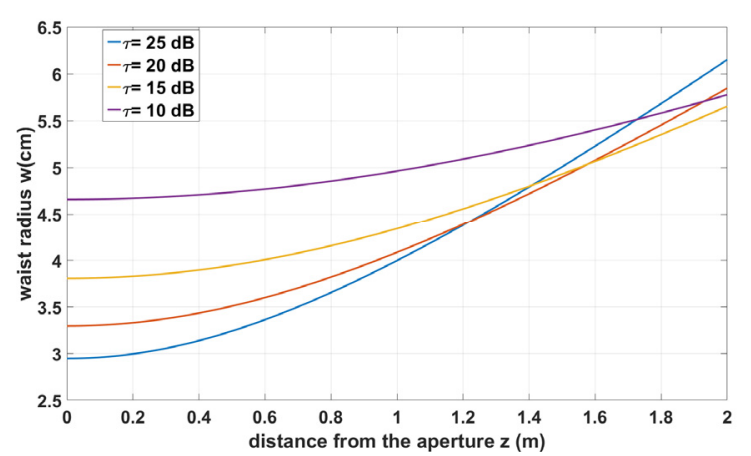

Fig. 6. Waist radius as a function of the distance to the aperture.

Once it has been shown that a reflector with an aperture of $10 \mathrm{~cm}$ diameter produces the desired illuminating spot on a target at $1.5 \mathrm{~m}$, the main challenge is to design and build an optimum reflector for the radar system. The geometry of the antenna system is shown in Fig. 7. The reflector is a 90 degrees offset reflector. The reason is to avoid blocking effects from the radar system. Notice that as shown in detail in Fig. 5 there are two sets of antennas, one for transmission and the other one for reception. It is clear that the arrays cannot be placed on the focus of the parabola and they are not contained on the focal plane. Notice that the focal plane of the reflector is in the xy plane and the feeding antennas are displaced on the $\mathrm{xz}$ plane.

In Fig. 8 the geometry of the parabola is shown. The focus is at the origin of the coordinate system and the parabolic curve is described in a parametric form as a function of the angle $\alpha$ as:

$$
\begin{gathered}
z=-f\left(1-\tan ^{2}\left(\frac{\alpha}{2}\right)\right) \\
y=2 f \tan \left(\frac{\alpha}{2}\right)
\end{gathered}
$$

Where $\mathrm{f}$ is the focal length. The offset reflector is constructed by intersecting the symmetric parabolic surface by a shifted cylinder with its axis parallel to the parabola symmetry axis. The diameter of the cylinder determines the aperture diameter. For a 90 degrees offset reflector yoffset is twice the focal length. For this application the parameter to optimize is the focal length of the reflector, since for the operational conditions the aperture diameter is set to $10 \mathrm{~cm}$ and to avoid the blockage produced by the radar a 90 degrees offset is desired. For a focus fed reflector the optimization of the focal length to maximize the antenna directivity is straightforward. In our case as it has been explained before the feeding antennas are not in focus.

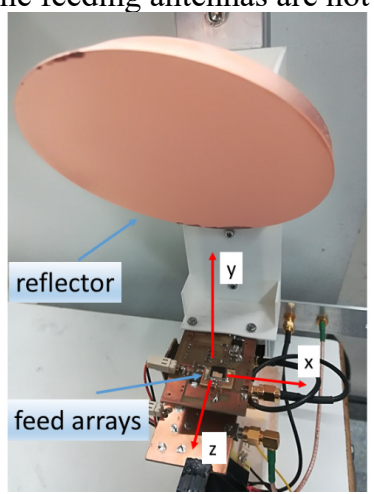

Fig. 7. Geometry of the antenna system.
Referring to the coordinate system that describes the reflector geometry of Fig. 7, the feeding antennas will be displaced 1.3 $\mathrm{mm}$ along the $\mathrm{x}$ axis and $0.6 \mathrm{~mm}$ along the $\mathrm{z}$ axis. This displacement will result in the maximum of the radiation pattern not pointing towards the broadside direction, the $\mathrm{z}$ axis, and a distortion on the radiation pattern. The consequence is that the maximum of radiation pattern for the transmitting and receiving antenna are not in the same direction. In [12] a detailed analysis of the effects of displacing the feed of the focus can be found. In the present design the only free parameter is the focal length of the reflector. A PO analysis of the reflector has been done. It is assumed that the reflector is in the far field of the feeds and the aperture fields on the reflector are numerically computed. The feed radiation pattern has been simulated assuming a uniformly fed 2 by 2 microstrip patch array with the dimensions shown in Fig. 5. From the aperture fields the radiation pattern is found.

Fig. 9 shows the evolution of the maximum directivity of the $10 \mathrm{~cm} 90$ degrees offset reflector with the feeder out of the focus and displaced $1.3 \mathrm{~mm}$ along the $\mathrm{x}$ axis and $0.6 \mathrm{~mm}$ along the $\mathrm{z}$ axis. One curve shows the value of the maximum directivity that does not occur at the broadside direction, which is achieved with a focal length of $40 \mathrm{~mm}$. Nevertheless, the goal is not to maximize the maximum directivity, but the value of the directivity on the broadside direction, that is $\theta=0$. In this case, the optimum focal length is $60 \mathrm{~mm}$. For this focal length the beams of the transmitting and receiving antennas overlap in the broadside direction with a directivity of $36.5 \mathrm{~dB}$.

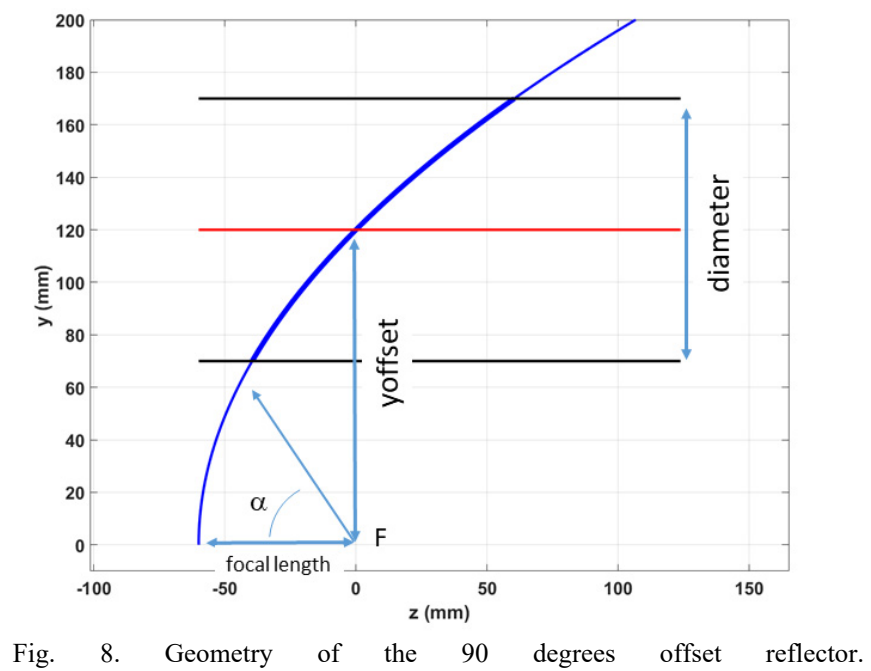




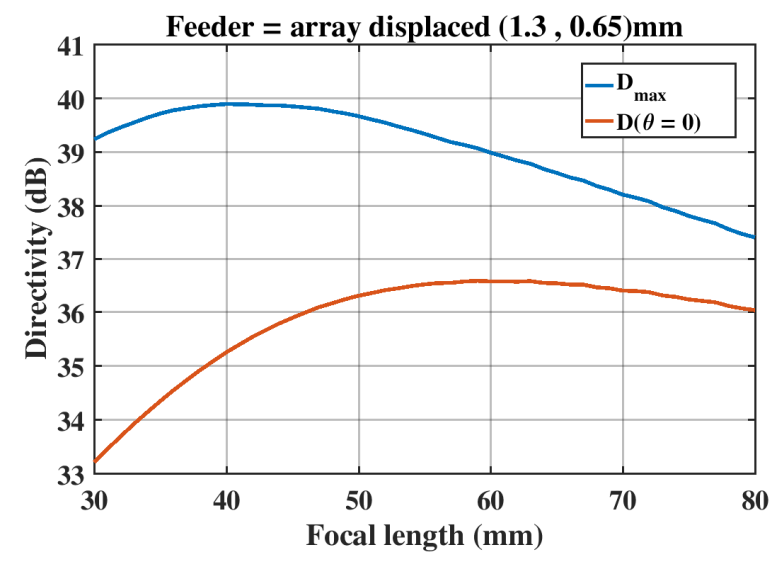

Fig. 9. Evolution of the $10 \mathrm{~cm} 90^{\circ}$ offset reflector directivity as a function of the focal length.

For the focal length of $60 \mathrm{~mm}$ the reflector has a spill over efficiency of 0.56 , an illumination efficiency of 0.91 and a polarization efficiency of 0.9 , so the overall efficiency is 0.46 or $3.14 \mathrm{~dB}$. Fig. 10 shows the simulated field distribution produced at distance $\mathrm{z}=1.5 \mathrm{~m}$ by the reflector when it is illuminated by each one of the feed antennas. In the figures the field has been normalized to the maximum and the circumference circumscribes the area that contains $90 \%$ of the energy. It is observed that the maximum of the field is not in the boresight direction and the diameter of the illuminated area is approximately $10 \mathrm{~cm}$. In Fig. 11 the product of the field produced by the transmitting and receiving antennas at a distance of $1.5 \mathrm{~m}$ is shown. It must be observed that as result of the design process when the combined transmitting and receiving performance is observed the maximum is at the broadside direction. Although the results are shown for a distance of $1.5 \mathrm{~m}$, the operation of the system can be extended to longer ranges. Fig. 12 shows the percentage of power contained in a circumference of $10 \mathrm{~cm}$ of diameter centered in the broadside direction as a function of the distance. It must be noticed, that the system is not focused in the near range at a given distance, but it is focused on the far field. Fig. 12 shows that extending the range of operation to 2 or $2.5 \mathrm{~m}$ results in a beam spreading of the collimated beam, but the system can still be operated.

In order to manufacture the reflector in a cost effective way it has been 3D printed. The reflector has been printed on a SIGMA 3D printer manufactured by $\mathrm{BCN} 3 \mathrm{D}$ and the process is described in [13]. It involves the printing of the reflector on a plastic material and afterwards the metallization of the surface by electroplating. The result can be seen in Fig. 7.

In order to assess the correct operation of the reflector antenna the set-up of Fig. 13 has been implemented. In the figure the radar with the reflector can be seen and at a distance of $1.5 \mathrm{~m}$ a two axis motorized linear system has been placed vertically. A low reflectivity Styrofoam support has been used to hold a $1 \mathrm{~cm}$ diameter pushpin acting as a target. The target has been scanned on a planar surface and the received signal by the radar has been recorded for each position. The measured signal is directly related with the field distributions on the measurement plane.
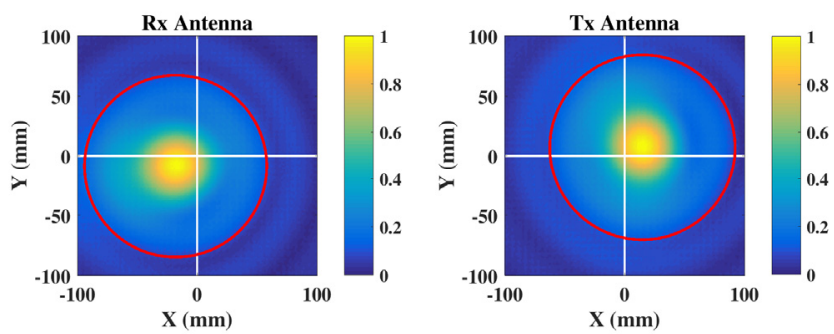

Fig. 10. Field distribution produced at distance $\mathrm{z}=1.5 \mathrm{~m}$ by each one of the feed antennas: TX Antenna and RX Antenna.

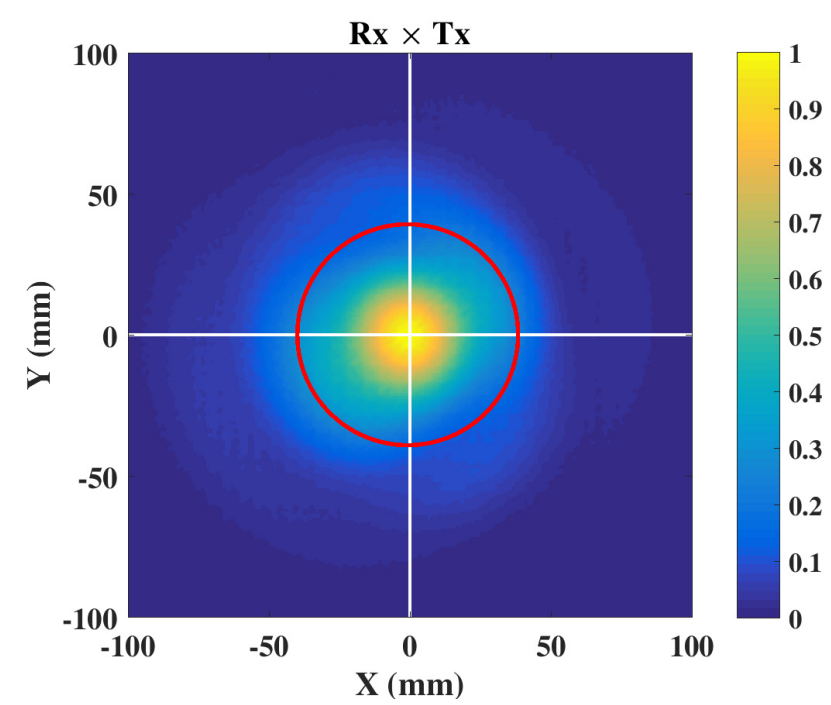

Fig. 11. Product of the normalized field distribution produced by the transmitting and receiving feed antennas at a distance of $1.5 \mathrm{~m}$.

Fig. 14 shows the measured results of the field distribution and they are compared with the theoretical ones. The theoretical ones correspond to the horizontal and vertical cut of the results shown in Fig. 11.

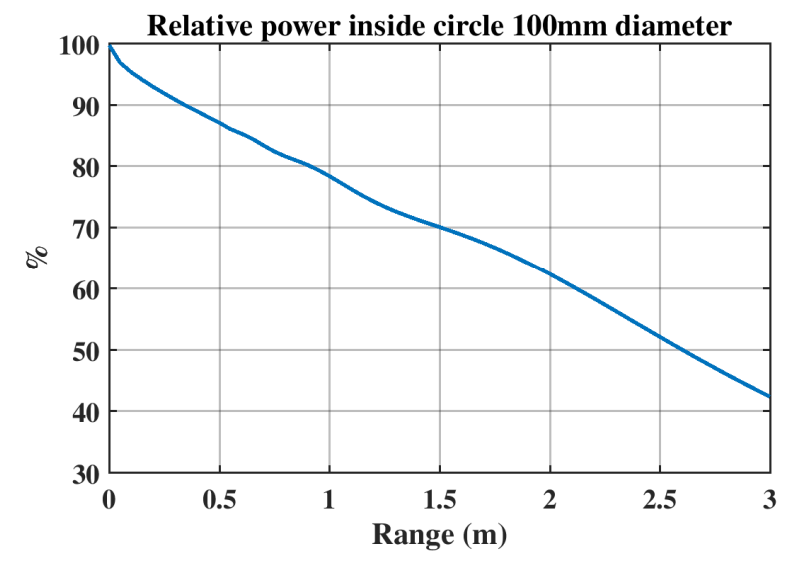

Fig. 12. Percentage of power contained in a circumference of $10 \mathrm{~cm}$ of diameter centered in the broadside direction as a function of the distance.

The results show the good performance of the reflector as compared to the simulated results, also the Gaussian beam behavior is clearly shown. In the vertical cut some deviations 
are observed from the theoretical values. Due to the large spillover losses of the reflector, one of the causes of this behavior can be a reflection in the supporting structure of the reflector.

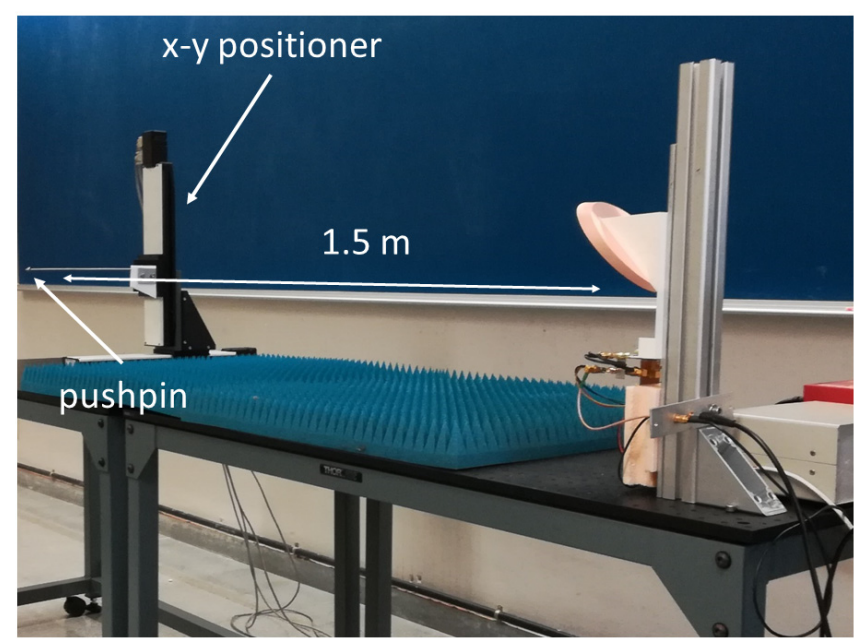

Fig. 13. Set-up in order to assess the correct operation of the reflector system, with the $\mathrm{x}-\mathrm{y}$ positioner frontal absorbing material removed.

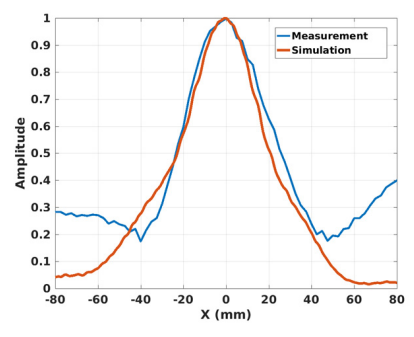

(a)

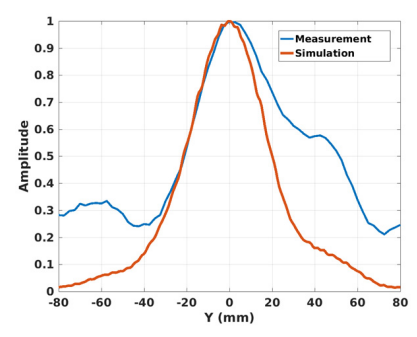

(b)
Fig. 14. Measured results of the field distribution for the set-up in Fig. 13 compared with the theoretical ones. (a) horizontal cut, (b) vertical cut.

\section{SYSTEM RESULTS}

Once the antenna system and the radar have been integrated it is necessary to perform a system evaluation. The first point is to assess potential health hazards. The nominal transmitted power is $\mathrm{Pt}=-3 \mathrm{dBm}$ with a reflector spillover efficiency of -2.5 $\mathrm{dB}$. As shown theoretically and in the measurements, the surface where the radiated power is concentrated can be approximated by a circle of $10 \mathrm{~cm}$ diameter. Therefore, the incident power density is $0.036 \mathrm{~W} / \mathrm{m}^{2}$. The ICNIRP reference levels for general public exposure in the frequency range between 2 and $300 \mathrm{GHz}$ is $10 \mathrm{~W} / \mathrm{m}^{2}$ [13]. The exposure level is more than 100 times below the reference level.

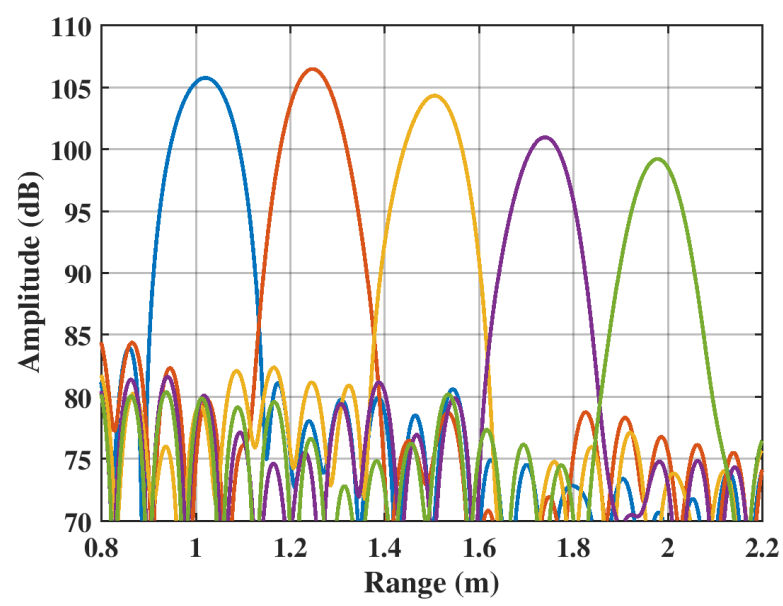

Fig 15. Range profiles obtained with a calibration sphere of $-34 \mathrm{dBsm}$ of RCS at different ranges.

The chip manufacturer does not provide nominal values for the receiver noise figure. In order to obtain the system sensitivity, the radar cross section (RCS) of a metal sphere of radius $\mathrm{a}=11 \mathrm{~mm}$ has been measured at a distance of $1,1.25,1.5$, 1.75 and $2 \mathrm{~m}$. At a frequency of $120 \mathrm{GHz}$ the monostatic RCS of the sphere is $\sigma=-34 \mathrm{dBsm}$. Fig. 15 shows the overlap of the measured signal with the calibration sphere at the five measurement distances. The result confirms the simulated beam collimation results shown in Fig. 12, as there is a certain reduction in the received signal due to the beam spreading, but much less as it would be expected in a far field condition. In order to estimate the signal to noise ratio (SNR) for the sphere at the position of $1.5 \mathrm{~m}, 1000$ measurements have been taken to estimate the average, $\bar{s}$ and the variance, $\sigma^{2}$ of the received signal. The SNR can be estimated as

$$
S N R=\frac{|\bar{s}|^{2}}{\sigma^{2}}
$$

The measurements have shown a SNR of $34.7,43.5$ and 53.2 $\mathrm{dB}$ with averaging factors of 1,10 and 100 . These results show a consistent behavior of an improvement of $10 \mathrm{~dB}$ in SNR when the averaging factor is increased by a factor of 10 , and they give an estimation of the system sensitivity. It can be expected that for a desired SNR of $10 \mathrm{~dB}$ and no averaging the sensitivity of the system is of the order of $-58 \mathrm{dBsm}$ at $1.5 \mathrm{~m}$.

It is also necessary to have an estimation of the RCS of a human torso. Fig. 16 shows the comparison of the measurements of the same dressed human torso at normal incidence and with an angle of $30^{\circ}$, compared to the $-34 \mathrm{dBsm}$ sphere. It can be observed that for normal incidence the received signal is about $10 \mathrm{~dB}$ above the one produced by the reference sphere. Further measurements should be done with different body types and incidence angles to have a more complete knowledge of value ranges of the expected received signal with the designed system. Nevertheless, with the measurements conducted so far a SNR better that $10 \mathrm{~dB}$ is clearly achievable at measurement ranges up to $2 \mathrm{~m}$. 


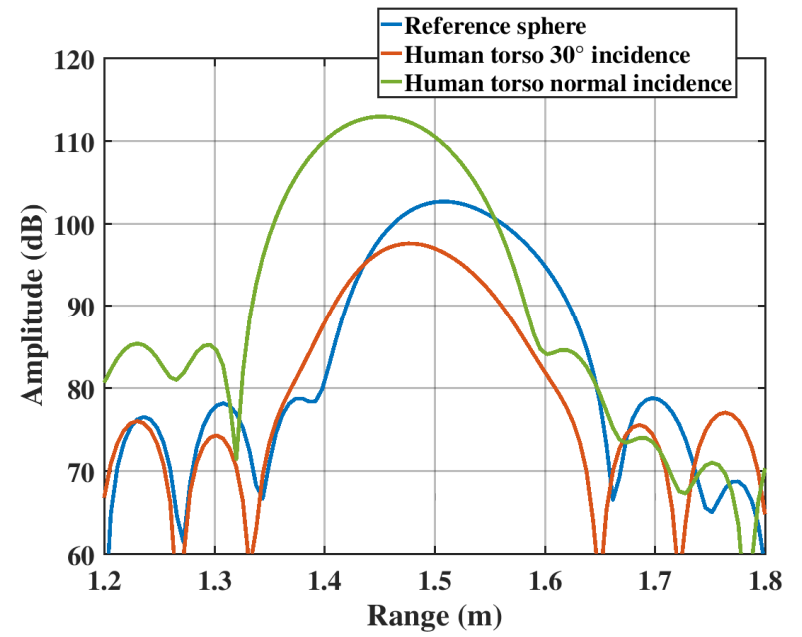

Fig. 16. Measurement of a human torso compared to the reference sphere of $34 \mathrm{dBsm}$.

To complete the assessment of the system it is necessary to evaluate its sensitivity to measure chest movements due to the heartbeat. In [15] chest movements between 0.5 and $1 \mathrm{~mm}$ due to heart beating are reported. Considering that the carrier frequency is $120 \mathrm{GHz}$ and the wavelength is $2.5 \mathrm{~mm}$, chest movements due to heart beating should produce measurable phase changes in the detected signal according to (7). Nevertheless, the limited signal to noise ratio in the measurements will cause errors in the estimation of the frequency and phase of the received signal and these errors will translate in range measurement errors. In [13] it is shown that the Cramer-Rao bound (CRB) for the variance of the range error due to a limited signal to noise ratio is:

$$
\operatorname{var}(R) \geq \frac{\lambda_{\text {start }}^{2}}{(2 \pi)^{2} N \cdot S N R}
$$

Where $\lambda_{\text {start }}$ is the wavelength at the starting frequency and $\mathrm{N}$ is the number of samples used to do the frequency and phase estimation through the FFT. For N=2048 samples and SNR of 10 the standard error of range has a lower bound of $2.7 \mu \mathrm{m}$. In [16] it is shown that in practice the standard error can be 6 or 7 times larger due to system imperfections. In any case even with a limited SNR of 10 the range error can be of the order of 30 $\mu \mathrm{m}$, which is one order of magnitude below the displacements that need to be measured.

The final test has been to measure the heart rate and the breathing rhythm using the radar. Fig. 17 shows on the top the experimental set-up. The test subject is sitting at approximately $1.5 \mathrm{~m}$ of the radar that is pointing at the upper part of the torso. The measurements have been conducted in a lab environment without any particular arrangement in order to reduce background reflections. In order to validate the measurement, a BIOPAC data logger equipped with a respiration belt transducer and photoplethysmogram (PPG) transducer able to measure respiration rate and heart beat rate has been used simultaneously with the radar acquisition.

Repeated trials observing the vital signs of a group of 8 volunteer students of both genders with ages between 22 to 24 years have been carried out to assess the robustness of the technique. Several data takes were acquired lying in supine (face up) position in 3 different breathing conditions: apnea, normal breathing, and forced abnormally deep and fast breathing. Radar observations were carried out from vertical or oblique directions with similar performance if the radar incidence is approximately perpendicular to the observed spot of the thorax. The respiratory signal was always observed and the associated frequency successfully obtained due to its large amplitude. In contrast the heart rate detection was found to be affected by the larger respiratory component. In apnea condition the heart rate was correctly detected in all $100 \%$ tests, in normal breathing condition the heart rate was correctly measured in $88 \%$ of the cases and in forced deep/fast breathing the heart rate was correctly detected in $50 \%$ of the tests indicating insufficient separation between respiratory and heart components of the measured motion

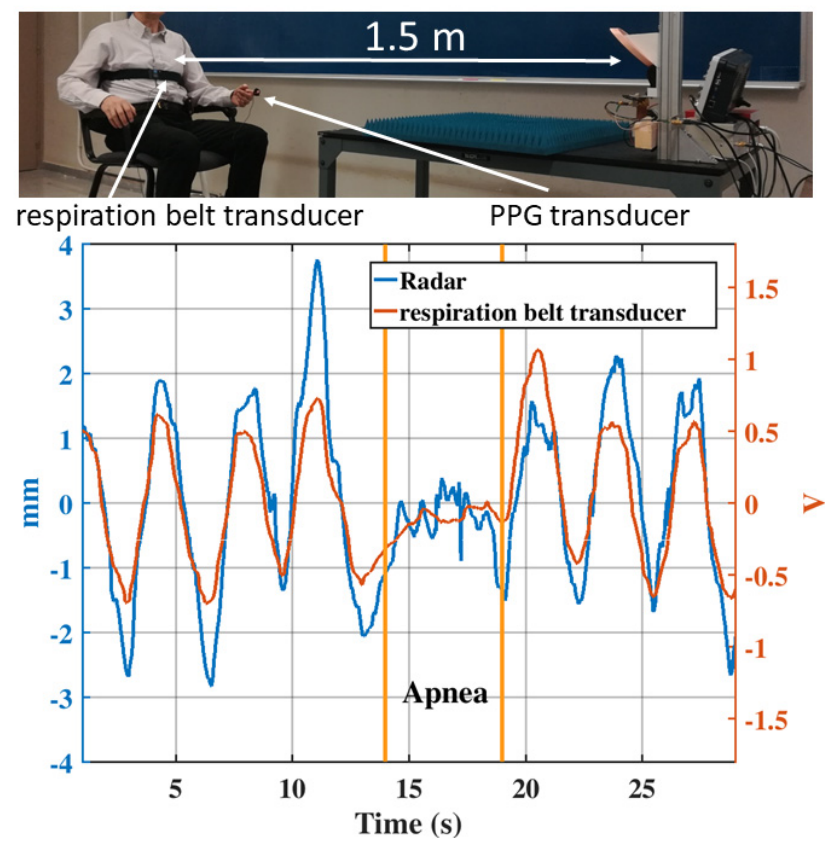

Fig. 17. Measurement set-up (top) and breathing rhythm measurement results (bottom).

Figure 17 (bottom) shows the chest displacements due to breathing measured with the radar and obtained from the phase term of (5). It is compared with the signal measured by the respiration belt transducer. Around the 15 second mark an apnea has been simulated and it is clearly seen the agreement between the recordings done with both sensors. The $2 \mathrm{~mm}$ chest movements due to the breathing are clearly measured. Since the movements due to the heartbeat are much smaller it is necessary to apply a band pass filter to remove the low frequency components associated to the breathing rhythm. Fig. 18 shows the results of a measurement when a band pass filter from 0.4 to $2 \mathrm{~Hz}$ is applied to the measured signal of Fig. 17. Chest movements of $0.2 \mathrm{~mm}$ are clearly measured and the correspondence with the registered signal by the PPG transducer is clearly shown. Notice that for a better comparison the two signals of Fig. 18 have been time aligned, as there is a delay from chest movements and readings by the PPG transducer on the finger. 


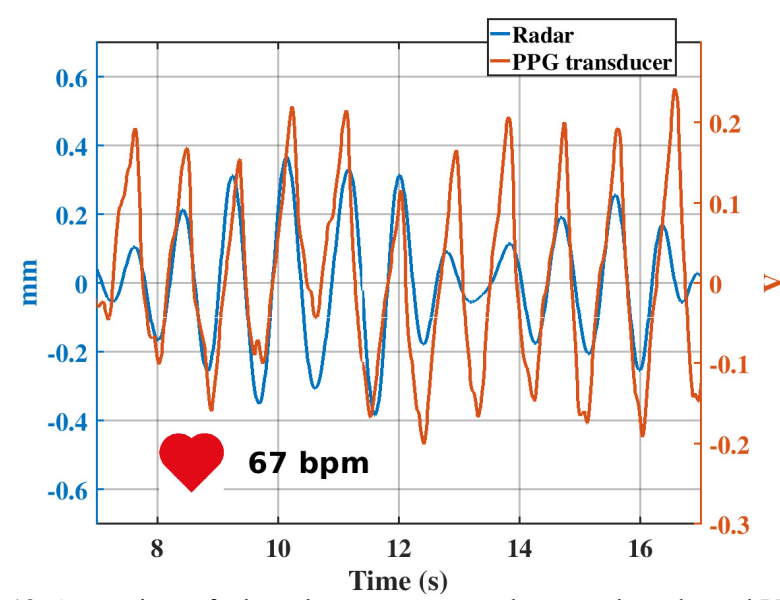

Fig. 18. Comparison of a heart beat measurement between the radar and PPG transducer.

\section{V. . CONCLUSIONS}

In this paper we have presented a non-contact and nonobstructive system for vital sign monitoring using a $120 \mathrm{GHz}$ FMCW radar. The results show that heart beat and breathing rate can be extracted accurately. The system can be operated in a clinical scenario due to its immunity to unwanted reflections. It has also been shown the necessary sensitivity to measure at distances of $1.5 \mathrm{~m}$. The availability of chipsets working at this frequency and the 3D printing of the parabolic reflector allows a relatively low-cost integration of a reduced dimensions system.

Once the sensitivity of the system has been demonstrated future work will encompass thorough clinical tests to assess the performance and accuracy when used in patients of different ages, gender and body types. Further work on the motion signal post-processing is required to improve the respiratory and cardiac component separation of the observed compound motion. In addition, the possibility to detect distinct anomalies such as: bradycardia and tachycardia or apnea and hyperventilation detection must be further investigated.

\section{REFERENCES}

[1] T.Naziyok,A. Zeleke,R, Röhrig,"Contactless Patient Monitoring for General Wards: A Systematic Technology Review", Studies in health technology and informatics 228:707-711

[2] Lin J. C., "Non-invasive microwave measurement of respiration," Proceedings of the IEEE, (1975), vol. 63, no.10, 1530

[3] Lin J. C., Kiernicki J., Kiernicki M., Wollschlaeger P. B., "Microwave Apexcardiography," Microwave Theory and Techniques, IEEE Transactions, (1979), vol. 27, no. 6, 618-620

[4] D. Obeid, S. Sadek, G. Zaharia and G. E. Zein, "Doppler radar for heartbeat rate and heart rate variability extraction," 2011 E-Health and Bioengineering Conference (EHB), Iasi, 2011, pp. 1-4.

[5] Pour Ebrahim, Malikeh, Majid Sarvi, and Mehmet Rasit Yuce. "A Doppler Radar System for Sensing Physiological Parameters in Walking and Standing Positions." Ed. Giancarlo Fortino et al. Sensors (Basel, Switzerland) 17.3 (2017): 485. PMC. Web. 7 Feb. 2018.

[6] Jussi Kuutti, Mikko Paukkunen, Miro Aalto, Pekka Eskelinen, Raimo E. Sepponen, "Evaluation of a Doppler radar sensor system for vital signs detection and activity monitoring in a radio-frequency shielded room", Measurement, Volume 68, 2015, Pages 135-142, ISSN 0263-2241, https://doi.org/10.1016/j.measurement.2015.02.048

[7] J.L.Eaves, E.K.Reedy (Eds.), "Principles of Modern Radar", Van Nostrand Reinhold, New York 1987
[8] J.A.Scheer, J.L.Kurtz (Eds.), "Coherent Radar Performance Estimation", Artech House, Nordwood, 1993

[9] A.Martínez, A.Aguasca, M.Lort, A.Broquetas, "Micrometric deformation imaging at W-Band with GBSAR", European Journal of Remote Sensing, 2016, 49: pp. 719-733.

[10] Silicon Radar, https://siliconradar.com/index_e.html

[11] N. J. McEwan and P. F. Goldsmith, "Gaussian beam techniques for illuminating reflector antennas," in IEEE Transactions on Antennas and Propagation, vol. 37, no. 3, pp. 297-304, March 1989. doi: 10.1109/8.18725

[12] W. Imbriale, P. Ingerson and W. Wong, "Large lateral feed displacements in a parabolic reflector," in IEEE Transactions on Antennas and Propagation, vol. 22, no. 6, pp. 742-745, Nov 1974. doi: 10.1109/TAP.1974.1140910

[13] J. Romeu, S. Blanch, N. Vidal, J. Lopez-Villegas and A. Aguasca, "Assessment of 3D Printing Technologies for Millimeter Wave Reflectors," in IEEE Antennas and Wireless Propagation Letters. doi: 10.1109/LAWP.2018.2857856

[14] ICNIRP. Guidelines for limiting exposure to time-varying electric, magnetic, and electromagnetic fields (up to $300 \mathrm{GHz}$ ). Health Phys 74:494-522; 1998

[15] Ø. Aardal, S. E. Hamran, T. Berger, Y. Paichard and T. S. Lande, "Chest movement estimation from radar modulation caused by heartbeats," 2011 IEEE Biomedical Circuits and Systems Conference (BioCAS), San Diego, CA, 2011, pp. 452-455. doi: 10.1109/BioCAS.2011.6107825

[16] S. Scherr, S. Ayhan, B. Fischbach, A. Bhutani, M. Pauli and T. Zwick, "An Efficient Frequency and Phase Estimation Algorithm With CRB Performance for FMCW Radar Applications," in IEEE Transactions on Instrumentation and Measurement, vol. 64, no. 7, pp. 1868-1875, July 2015. doi: 10.1109/TIM.2014.2381354

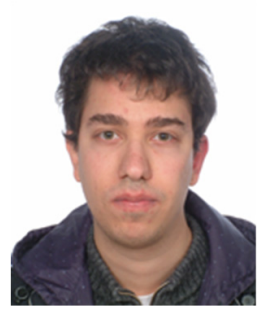

Arnau Prat (S'14-M'18) was born in Girona, Spain, in 1993. He received the B.S. degree in electronics systems engineering and the M.S. degree in telecommunications engineering from the Polytechnic University of Catalonia (UPC), Barcelona, in 2015 and 2017, respectively. From September 2016 to February 2017, he was a Research Assistant with the Department of Signal Theory and Communications, UPC, where he worked on a terahertz radar system. More recently he has been a visiting student at the department of Mechanical and Aerospace Engineering at Cornell University.

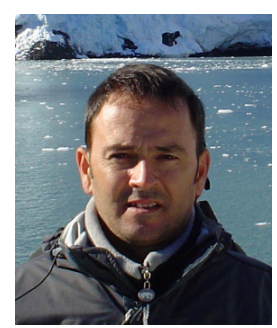

Albert Aguasca (S'90-M'94) was born in Barcelona, Spain, in 1964. He received the M.Sc. and Ph.D. degrees in telecommunication engineering from the Universitat Politecnica de Catalunya (UPC), Barcelona, in 1989 and 1993, respectively. Since 1995, he has been an Associate Professor with the School of Telecommunications Engineering, UPC. His teaching activities involve radio-frequency and microwave circuits for communications and radio navigation systems. He has authored or coauthored more than 40 papers on microwave synthetic aperture radar (SAR), radiometer systems, and microwave circuits. His research interests include the design and development of SAR and microwave radiometer systems for unmanned aerial vehicle platforms. 


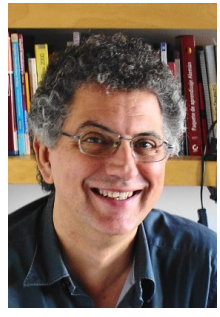

Jordi Romeu (F'12) was born in Barcelona, Spain, in 1962. He received the Ingeniero de Telecomunicación and Doctor Ingeniero de Telecomunicacíon degree from the Universitat Politècnica de Catalunya (UPC), Barcelona, Spain, in 1986 and 1991, respectively. He has been with the Electromagnetic and Photonic Engineering Group, Signal Theory and Communications Department, UPC, since 1985, where he is currently a full Professor, where he is involved in the research of antenna nearfield measurements, antenna diagnostics, and antenna design. He joined the Antenna Laboratory, University of California, Los Angeles, CA, USA, in 1999, as a Visiting Scholar, under the North Atlantic Treaty Organization Scientific Program Scholarship. In 2004, he joined the University of California, Irvine, CA, USA. He has authored 50 refereed papers in international journals and 50 conference proceedings and holds several patents. Dr. Romeu received the Grand Winner of the European IT Prize by the European Commission, for his contributions in the development of fractal antennas in 1998.

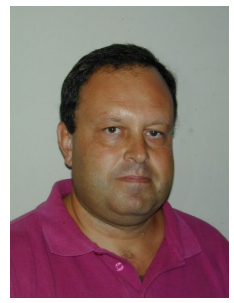

Sebastián Blanch was born in Barcelona, Spain, in 1961. He received the Ingeniero and Doctor Ingeniero degrees in Telecommunication Engineering, both from the Polytechnic University of Catalonia (UPC), Barcelona, Spain, in 1989 and 1996, respectively. In 1989, he joined the Electromagnetic and Photonics Engineering Group of the Signal Theory and Communications Department. Currently, he is Associate Professor at UPC. His research interests are antenna near field measurements, antenna diagnostics, and antenna design.

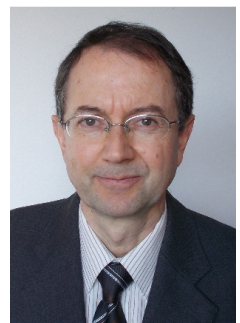

Antoni Broquetas (S'84-M'90) was born in Barcelona, Spain, in 1959. He received the Ingeniero degree in telecommunication engineering and the Doctor Ingeniero degree in telecommunications engineering (microwave tomography) from the Universitat Politècnica de Catalunya (UPC), Barcelona, Spain, in 1985 and 1989, respectively. In 1986, he was a Research Assistant with Portsmouth Polytechnic, Portsmouth, U.K., involved in propagation studies. In 1987, he joined the Department of Signal Theory and Communications (TSC), UPC. From 1998 to 2002, he was a Subdirector of Research with the Institute of Geomatics, Barcelona, Spain. From 1999, he is a Full Professor with UPC involved in research on radar imaging and remote sensing. From 2003 to 2006, he was a Director of the TSC, UPC. He has authored more than 150 papers on microwave tomography, Radar, ISAR and SAR systems, SAR processing, and interferometry. 